\title{
RINGS SATISFYING MONOMIAL CONSTRAINTS
}

\author{
MOHAN S. PUTCHA ${ }^{1}$ AND ADIL YAQUB
}

\begin{abstract}
The following theorem is proved: Suppose $R$ is an associative ring and suppose $J$ is the Jacobson radical of $R$. Suppose that for all $x_{1}, \cdots, x_{n}$ in $R$, there exists a word $w_{x_{1}}, \cdots, x_{n}\left(x_{1}, \cdots, x_{n}\right)$, depending on $x_{1}, \cdots, x_{n}$, in which at least one $x_{i}$ (i varies) is missing, and such that $x_{1} \cdots x_{n}=w_{x_{1}}, \cdots, x_{n}\left(x_{1}, \cdots, x_{n}\right)$. Then $J$ is a nil ring of bounded index and $R / J$ is finite. It is further proved that a commutative nil semigroup satisfies the above identity if and only if it is nilpotent.
\end{abstract}

In this paper, we investigate the structure of an associative ring $R$ with the property that, for all $x_{1}, \cdots, x_{n}$ in $R, x_{1} \cdots x_{n}=w_{x_{1}, \cdots x_{n}}\left(x_{1}, \cdots, x_{n}\right)$, where $w$ is a word, depending on $x_{1}, \cdots, x_{n}$, and where some $x_{i}(i$ varies) is missing in $w$. For such a ring $R$, we prove the Jacobson radical $J$ is a nil ring of bounded index. We also show that $R / J$ is finite. Finally, we show that a commutative nil semigroup satisfies the above identity if and only if it is nilpotent.

In preparation for the proofs of the main results, we first introduce

Definition 1. Let $n$ be a positive integer, and let $S$ be a semigroup. We say that $S$ is a $\beta_{n}$-semigroup if, for all $x_{1}, \cdots, x_{n}$ in $S$, there exists a word $w_{x_{1}}, \cdots, x_{n}\left(x_{1}, \cdots, x_{n}\right)$ consisting of a product of the $x_{i}$ 's with some $x_{j}$ ( $j$ varies) missing, such that $x_{1} \cdots x_{n}=w_{x_{1}, \cdots, x_{n}}\left(x_{1}, \cdots, x_{n}\right)$. A ring $R$ is called a $\beta_{n}$-ring if its multiplicative semigroup is a $\beta_{n}$-semigroup.

LEMMa 1. Let $S$ be a finite semigroup or a nilpotent semigroup. Then $S$ is a $\beta_{n}$-semigroup, for some $n$.

Proof. First, suppose $S$ is finite, of order $n$, and suppose $x_{1}, \cdots, x_{n+1}$ are any elements of $S$. Then $x_{i}=x_{j}$ for some $i>j$, and hence

$$
\begin{aligned}
x_{1} \cdots x_{n+1} & =x_{1} \cdots x_{j} \cdots x_{i-1} x_{j} x_{i+1} \cdots x_{n+1} \\
& =w\left(x_{1}, \cdots, x_{i-1}, x_{i+1}, \cdots, x_{n+1}\right) .
\end{aligned}
$$

Thus $S$ is a $\beta_{n+1}$-semigroup. Next, suppose $S$ is nilpotent, say, $S^{m}=(0)$.

Presented to the Society, January 18, 1972: received by the editors August 10, 1971 and, in revised form, October 6, 1971.

AMS (MOS) subject classifications (1970). Primary 16A38, 16A48; Secondary $20 \mathrm{M} 10$.

${ }^{1}$ The author was supported by a National Science Foundation Graduate Fellowship.

(c) American Mathematical Society 1973 
Then, for all elements $x_{1}, \cdots, x_{m+1}$ of $S$, we have

$$
x_{1} \cdots x_{m+1}=0=x_{1} \cdots x_{m} .
$$

Thus $S$ is a $\beta_{m+1}$-semigroup, and the lemma is proved.

We now pause to give an example of a $\beta_{n}$-ring which is not of the type described in Lemma 1 . To this end, let $R_{0}$ be an infinite field of characteristic 2 , and let

$$
R=\left\{\left(\begin{array}{ll}
a & u \\
0 & 0
\end{array}\right) \mid a \in G F(2), u \in R_{0}\right\} .
$$

It is readily verified that $R$ is a $\beta_{3}$-ring. Indeed, $x_{1} x_{2} x_{3}=x_{2} x_{3}$ if

while $x_{1} x_{2} x_{3}=x_{1} x_{2}$ if

$$
x_{1}=\left(\begin{array}{ll}
1 & u \\
0 & 0
\end{array}\right)
$$

$$
x_{1}=\left(\begin{array}{ll}
0 & u \\
0 & 0
\end{array}\right)
$$

Moreover, $R$ is neither finite nor nilpotent and, in fact, $R$ is not isomorphic to any finite direct sum of finite or nilpotent rings. (An example of an infinite $\beta_{n}$-group is given in Remark 1 below.)

Lemma 2. Let $S$ be a $\beta_{n}$-semigroup. Then any subsemigroup of $S$ and any homomorphic image of $S$ are also $\beta_{n}$-semigroups.

This follows at once from the definition.

Lemma 3. Let $S$ be a $\beta_{n}$-semigroup. Then there exists a fixed positive integer $k$ with the property that, for any $a$ in $S$, there is an integer $l>k$ such that $a^{k}=a^{l}$.

Proof. Let $q_{1}, \cdots, q_{n}$ be the first $n$ positive primes, and let

$$
m_{i}=\left(q_{1} \cdots q_{n}\right) / q_{i} \quad(i=1, \cdots, n) .
$$

Let $k=m_{1}+\cdots+m_{n}$. Now, since $S$ is a $\beta_{n}$-semigroup, there exists a word $w\left(a^{m_{1}}, \cdots, a^{m_{n}}\right)$, with some $a^{m_{j}}$ missing in the word $w$, such that

$$
a^{k}=\left(a^{m_{1}}\right) \cdots\left(a^{m_{n}}\right)=w\left(a^{m_{1}}, \cdots, a^{m_{n}}\right) \equiv a^{l} .
$$

Since $a^{m_{j}}$ is missing from $w$, it follows, by (1), that $q_{j}$ divides $l$. But, by (1) again, $q_{j}$ does not divide $k$, and hence $l \neq k$. Now, if in (2), $l<k$, then by iterating in (2), we can make $l>k$. This proves the lemma.

Notation 1. Let $Z^{+}$denote the set of all positive integers, and let $D(s)=\left\{m \mid m \in Z^{+}, m\right.$ divides $\left.s\right\} ; \quad P(s)=\{m \mid m \in D(s), m$ is prime $\}$. 
If $S$ is any nonempty subset of $Z^{+}$, then

$$
D(S)=\bigcup_{s \in S} D(s) \text { and } P(S)=\bigcup_{s \in S} P(s) .
$$

LEMMA 4. Let $S$ be any infinite set of positive integers and let $P(S)$ be finite. Then there exists $q \in P(S)$ such that $q^{i} \in D(S)$ for all $i \in Z^{+}$.

Proof. If the lemma were false, then $D(S)$ would be finite, since $P(S)$ is finite. But $D(S)$ is infinite, since $D(S) \supseteq S$.

In preparation for the proof of the next lemma, we first state the following two well-known results. The proof of Lemma A appears in [4, p. 164], while the proof of Lemma B appears in [6, p. 2].

LemMA A. Let $a$ be an integer and $n$ an integer $>1$. Let $\Phi_{n}(x)$ denote the cyclotomic polynomial of order $n[5, \mathrm{p} .60]$. If a prime number $p$ divides $\Phi_{n}(a)$, then either $p \mid n$ or $n \mid p-1$.

LEMMA B. Let $l$ be a prime number and $a$ an integer $>1$. Then $\Phi_{l^{v}}(a)$ is a multiple of $l$ if and only if $a \equiv 1 \bmod l$. If $a \equiv 1 \bmod l$, then the l-order of $\Phi_{l^{v}}(a)$ is equal to 1 except in the case where $l=2, \nu=1$, and $a \equiv 3 \bmod 4$. For any $\nu, \mu>0, \nu \neq \mu$, G.C.D. $\left(\Phi_{l^{\mu}}(a), \Phi_{l^{v}}(a)\right)=1$ or $l$.

COROLlARY. Let $l$ be a prime number, $a$ an integer $>1$, and suppose $a \equiv 1 \bmod l$. Then there exist infinitely many prime numbers dividing $\Phi_{l^{v_{1}}}(a), \Phi_{l^{v_{2}}}(a), \cdots$ for any increasing sequence $v_{1}, v_{2}, \cdots \rightarrow \infty$.

We are now in a position to prove

LEMMA 5. Let $a$ be an integer $>1$ and let

$$
T=\left\{k_{i} \mid i \in Z^{+}\right\}
$$

be a strictly increasing sequence of positive integers such that $k_{i}$ divides $k_{i+1}$ for each $i$. Let

$$
S=\left\{a^{k_{i}}-1 \mid i \in Z^{+}\right\}
$$

Then $P(S)$ is infinite.

Proof. We distinguish two cases.

Case 1. $P(T)$ is infinite. Let $E_{1}=\left\{p_{1}, \cdots, p_{l}\right\}$ be a finite set of prime numbers, and $k_{1}^{\prime}, k_{2}^{\prime}, \cdots$ be the series of integers such that

$$
k_{n}=p_{1}^{c_{1}, n} \cdots p_{l}^{c_{l, n}} k_{n}^{\prime} \quad\left(k_{n}^{\prime}, p_{1} \cdots p_{l}\right)=1 .
$$

Then, since $P(T)$ is infinite, $\lim _{n \rightarrow \infty} k_{n}^{\prime}=\infty$. Hence, for any $n$ with $k_{n}^{\prime} \geqq \max \left(p_{1}, \cdots, p_{l}\right)$, we conclude, using Lemma $\mathrm{A}$, that $p_{1}, \cdots, p_{l}$ do not divide $\Phi_{k_{n}{ }^{\prime}}(a)$. Moreover, since $k_{n}^{\prime}$ divides $k_{n}, \Phi_{k_{n}{ }^{\prime}}(a)$ divides $a^{k_{n}}-1$. Thus, if $p$ is any prime divisor of $\Phi_{k_{n}}(a)$, then $p$ divides $a^{k_{n}}-1$ and, clearly, $p \neq p_{i}(i=1, \cdots, l)$. Hence $P(S)$ is infinite. 
Case 2. $P(T)$ is finite. In this case, we argue by contradiction. Thus suppose $P(S)$ is finite. Since $P(T)$ is finite, it follows by Lemma 4 that there exists $l \in P(T)$ such that

$$
l^{j} \in D(T) \text { for all } j \in Z^{+} .
$$

Now, let

$$
S^{\prime}=\left\{a^{l^{j}}-1 \mid j \in Z^{+}\right\} .
$$

Then, by (5) and (3), $l^{j} \mid k_{i}$ for some $k_{i}$, and hence $a^{l^{j}}-1 \mid a^{k_{i}}-1$. Therefore $P\left(S^{\prime}\right) \subseteq P(S)$, and hence $P\left(S^{\prime}\right)$ is finite. Thus, there exists $k \in Z^{+}$such that

$$
P\left(a^{l^{k}}-1\right)=P\left(a^{l^{k+1}}-1\right) .
$$

Moreover, observe that $a^{k^{k+1}}-1=\left(a^{l^{k}}-1\right) w$, where

$$
w=\left(a^{l^{k}}\right)^{l-1}+\cdots+\left(a^{l^{k}}\right)+1 .
$$

Now, let $t \in P(w)$. Then $t \in P\left(a^{l^{k+1}}-1\right)=P\left(a^{l^{k}}-1\right)$, and hence $a^{l^{k}} \equiv 1 \bmod t$. Combining this with (8), we get $0 \equiv w \equiv l \bmod t$, and hence $t \mid l$. Therefore $t=l$. Moreover, by Fermat's Little Theorem, $a^{l} \equiv a \bmod l$ and hence $a^{l^{k}} \equiv a \bmod l$. Combining this with $a^{l^{k}} \equiv 1 \bmod t(=l)$, we obtain $a \equiv 1 \bmod l$. Hence, by the corollary to Lemma $\mathrm{B}$, there exists infinitely many prime numbers dividing $\Phi_{l^{v_{1}}}(a), \Phi_{l^{v_{2}}}(a), \cdots$ for any increasing sequence $v_{1}, v_{2}, \cdots \rightarrow \infty$. Moreover, since $\Phi_{l^{v_{1}}}(a)$ divides $a^{l_{v_{1}}}-1$, and recalling (6), we conclude that $P\left(S^{\prime}\right)$ is infinite. But, as we have shown above, $P\left(S^{\prime}\right)$ is finite. This contradiction proves the lemma.

We call a field $F$ periodic if, for every $x$ in $F$, we have $x^{m}=x^{n}$ for some positive integers $m, n, m \neq n$.

THeorem 1. Suppose that $F$ is a $\beta_{n}$-field. Then $F$ is finite.

Proof. Suppose $F$ is infinite. Since, by Lemma $3, F$ is periodic, $F$ has a prime characteristic $p$. Moreover, the subfield $\langle x\rangle$ generated by a single element $x$ in $F$ is finite, and hence

$$
x^{p^{k}}=x \text { for some positive integer } k=k(x) .
$$

Now, for each $j \in Z^{+}$, define the finite field

$$
F_{j}=\left\{x \mid x \in F, x^{p^{j !}}=x\right\}
$$

Then, in view of (9) and (10), we have (since if $x \in F$ satisfies (9), then $\left.x \in F_{k}\right)$

$$
F_{1} \subseteq F_{2} \subseteq F_{3} \subseteq \cdots ; \text { each } F_{i} \text { is a subfield of } F ; \bigcup_{i \in Z^{+}} F_{i}=F .
$$


Now, since $F$ is infinite, we can find a subsequence of (11) such that

$$
F_{i_{1}} \varsubsetneqq F_{i_{2}} \varsubsetneqq F_{i_{3}} \subsetneq \cdots \text {, and again } \bigcup_{k \in Z^{+}} F_{i_{k}}=F \text {. }
$$

Moreover, the order of $F_{i_{\sigma}}=p^{k_{\sigma}}\left(\sigma \in Z^{+}\right)$. Next, let

$$
S=\left\{p^{k_{\sigma}}-1 \mid \sigma \in Z^{+}\right\} .
$$

Then, as is well known, $k_{\sigma} \mid k_{\sigma+1}$ for each $\sigma \in Z^{+}$, and $k_{\sigma}<k_{\sigma+1}$, by (12). Hence, by Lemma $5, P(S)$ is infinite, and there, therefore, exist $n$ distinct primes $q_{1}, \cdots, q_{n}$ in $P(S)$. Thus, there exists $m_{j} \in Z^{+}$such that

$$
q_{j} \in P\left(p^{k_{m_{j}}}-1\right) \quad(j=1, \cdots, n) .
$$

Now, since the nonzero elements of the field $F_{i_{m_{j}}}$ form a multiplicative group of order $p^{k_{m_{j}}}-1$, and since the prime $q_{j} \mid p^{k_{m_{j}}}-1$, it follows, by Cauchy's Theorem, that there exists $x_{j} \in F_{i_{m_{j}}}(\subseteq F)$ such that

$$
\text { order of } x_{j}=q_{j} \quad(j=1, \cdots, n) .
$$

Moreover, since $F$ is a $\beta_{n}$-field, there exists a word $w\left(x_{1}, \cdots, x_{n}\right)$, with some $x_{j}$ missing, such that

$$
x_{1} \cdots x_{n}=w\left(x_{1}, \cdots, x_{n}\right) .
$$

But, by (14), the order of $x_{1} \cdots x_{n}=q_{1} \cdots q_{n}$, while the order of $w\left(x_{1}, \cdots, x_{n}\right) \leqq\left(q_{1} \cdots q_{n}\right) / q_{j}$ (since $x_{j}$ does not appear in $w$ ), a contradiction. This contradiction proves the theorem.

Corollary 1. Any $\beta_{n}$-division ring $D$ is finite.

Proof. By Lemma 3, $D$ is periodic, and hence, for any $x \in D$, we have $x^{m}=x^{k}$, for some $m, k, m \neq k$. Therefore, $x^{r(x)}=x$ for some $r(x)>1$, and hence by Jacobson's Theorem [2, p. 217], $D$ is a field. The corollary now follows at once from Theorem 1.

Corollary 2. Any $\beta_{n}$-primitive ring $R$ is finite.

PRoof. By Jacobson's Density Theorem [2, p. 33], either $R \cong D_{m}$, where $D_{m}$ is a complete matrix ring over a division ring $D$, in which case we are done by Corollary 1 and Lemma 2, or $D_{n+1}$ is a homomorphic image of some subring $R_{0}$ of $R$. Now, by Lemma $2, D_{n+1}$ is a $\beta_{n}$-ring also. Let $x_{i} \in D_{n+1}$ be the matrix with 1 in the $(i, i+1)$ position and zero elsewhere:

$$
x_{i}=E_{i, i+1} \quad(i=1, \cdots, n) .
$$

It is readily verified that

$$
x_{1} \cdots x_{n}=E_{1, n+1} \neq 0 \text {, and } x_{i} x_{j} \neq 0 \text { if and only if } j=i+1 .
$$

Since $D_{n+1}$ is a $\beta_{n}$-ring, we have

$$
x_{1} \cdots x_{n}=w\left(x_{1}, \cdots, x_{n}\right) \text {; some } x_{j} \text { missing in } w .
$$


In view of (17) and (18), we obtain $w\left(x_{1}, \cdots, x_{n}\right) \neq 0$ and thus (see (17)) (19) $w\left(x_{1}, \cdots, x_{n}\right)=x_{\sigma} x_{\sigma+1} x_{\sigma+2} \cdots x_{\sigma+\tau} ; \quad \sigma \neq 1$ or $\sigma+\tau \neq n$. Hence, by (16)-(19), we obtain $E_{1, n+1}=E_{\sigma, \sigma+\tau+1}$, and thus $\sigma=1$ and $\sigma+\tau=n$, which contradicts (19). This proves the corollary.

Next, we prove the following:

Lemma 6. Let $R_{1}, \cdots, R_{n}$ be associative rings with identities. Then the direct sum $R_{1} \dot{+} \cdots+R_{n}$ is not a $\beta_{n}$-ring.

Proof. Suppose that $R_{1} \dot{+} \cdots+R_{n}$ is a $\beta_{n}$-ring, and define

$$
x_{i}=(1,1, \cdots, 1,0,1,1, \cdots, 1) \text {, }
$$

0 is in the $i$ th position $(i=1, \cdots, n)$.

Since $R_{1}+\cdots+R_{n}$ is a $\beta_{n}$-ring, there exists a word $w\left(x_{1}, \cdots, x_{n}\right)$ such that

$$
x_{1} \cdots x_{n}=w\left(x_{1}, \cdots, x_{n}\right) \text {; some } x_{j} \text { missing in } w \text {. }
$$

Comparing the $j$ th coordinates of both sides of (21), we obtain $0=1$ (since $x_{j}$ is missing in $w$ ), a contradiction. This proves the lemma.

LEMMA 7. Let $R$ be an associative ring, and let $I_{1}, I_{2}$ be ideals of $R$ with $I_{1} \ddagger I_{2}$ and $R / I_{2}$ simple. Then $R / I_{1} \cap I_{2} \cong R / I_{1}+R / I_{2}$.

Proof. Since $R / I_{2}$ is simple and $I_{1} \ddagger I_{2}, I_{1}+I_{2}=R$, and hence, by the second isomorphism theorem,

$$
R / I_{1} \cap I_{2} \cong I_{1} / I_{1} \cap I_{2}+I_{2} / I_{1} \cap I_{2} \cong R / I_{2}+R / I_{1}
$$

We are now in a position to prove the following fundamental

THEOREM 2. Let $R$ be a semisimple ring. Then $R$ is a $\beta_{n}$-ring, for some $n$, if and only if $R$ is finite.

Proof. Suppose that $R$ is a $\beta_{n}$-ring and suppose $R$ is infinite. We shall show that this leads to a contradiction. Since $R$ is semisimple, there exist ideals $I_{\alpha}(\alpha \in \Omega)$ of $R$ such that [2, p. 14] $\bigcap_{\alpha \in \Omega} I_{\alpha}=(0)$, and each $R / I_{\alpha}$ is primitive.

By Corollary 2 and Lemma 2 , each $R / I_{\alpha}$ is finite, and hence [2, p. 33] each of the primitive rings $R / I_{\alpha}$ is a complete matrix ring over a finite field. Thus each $R / I_{\alpha}$ is a simple ring with identity. Next, choose $\alpha_{1} \in R$, and having chosen $\alpha_{1}, \cdots, \alpha_{k}$ so that $\sum_{i=1}^{\cdot k} R / I_{\alpha_{i}} \cong R / \bigcap_{i=1}^{k} I_{\alpha_{i}}$, choose $\alpha_{k+1} \in \Omega$ such that $\bigcap_{i=1}^{k} I_{\alpha_{i}} \neq I_{\alpha_{k+1}}$. That such $\alpha_{k+1}$ can always be so chosen is proved as follows: Suppose no such $\alpha_{k+1}$ exists. Then $(0)=\bigcap_{\alpha \in \Omega} I_{\alpha}=\bigcap_{i=1}^{k} I_{\alpha_{i}}$, and hence

$$
R \cong R / \bigcap_{i=1}^{k} I_{\alpha_{i}} \cong \sum_{i=1}^{k} R / I_{\alpha_{i}}
$$


Thus $R$ is finite, a contradiction. This contradiction shows that there exists $\alpha_{k+1} \in \Omega$ such that $\bigcap_{i=1}^{k} I_{\alpha_{i}} \neq I_{\alpha_{k+1}}$. Hence, by Lemma 7,

$$
R / \bigcap_{i=1}^{k+1} I_{\alpha_{i}} \cong R / \bigcap_{i=1}^{k} I_{\alpha_{i}}+R / I_{\alpha_{k+1}} \cong \sum_{i=1}^{k+1} R / I_{\alpha_{i}}
$$

In particular, we obtain

$$
\sum_{i=1}^{n} R / I_{\alpha_{i}} \cong R / \bigcap_{i=1}^{n} R / I_{\alpha_{i}}
$$

Now, by Lemma 2, $R / \bigcap_{i=1}^{n} R / I_{\alpha_{i}}$ is a $\beta_{n}$-ring, and hence $\sum_{i=1}^{n} R / I_{\alpha_{i}}$ is a $\beta_{n}$-ring also, which contradicts Lemma 6 . This contradiction shows that $R$ is finite. The converse part follows at once from Lemma 1 .

Combined with Lemma 3, we easily obtain

COROLlaRY 3. Let $R$ be an associative $\beta_{n}$-ring with Jacobson radical $J$. Then $J$ is a nil ring of bounded index, and $R / J$ is finite.

In the above corollary, it follows [1, p. 28] that the Jacobson radical is locally nilpotent. It is not known to the authors whether the Jacobson radical must be nilpotent. However we have

THEOREM 3. Let $S$ be a commutative nil semigroup. Then $S$ is a $\beta_{n^{-}}$ semigroup, for some $n$, if and only if $S$ is nilpotent.

Proof. Let $S$ be a $\beta_{n}$-semigroup. By Lemma $3, x^{k}=0$ for all $x \in S$. So there exists a least positive integer $q$ for which a positive integer $m$ exists such that, for all $a_{1}, \cdots, a_{m} \in S$,

$$
a_{1}^{q} \cdots a_{m}^{q}=0 \quad(q \text { minimal }) .
$$

We now assume that $q>1$ and obtain a contradiction. Let $a_{1}, \cdots, a_{m n} \in S$, and for each $i, 0 \leqq i \leqq n-1$, define $x_{i+1}=a_{i m+1}^{q-1} \cdots a_{i m+m}^{q-1}$. Then, since $S$ is commutative and $q \geqq 2$, we have by (22), $x_{i+1}^{2}=0$ for each $i$. Since $S$ is a $\beta_{n}$-semigroup,

$$
x_{1} \cdots x_{n}=w\left(x_{1}, \cdots, x_{n}\right)=w \text {, some } x_{j} \text { missing in } w .
$$

Now, if some $x_{i}$ appears twice in the word $w$, then by (23), $x_{1} \cdots x_{n}=0$. Otherwise, since $S$ is commutative and $x_{j}$ does not appear in $w$, we can write $x_{1} \cdots x_{n} \equiv w v$, where $v$ is a product of at least one $x_{i}$. Hence, by (23), we have $w v=w$. Therefore, $w=w v=w v^{k}=0$, and hence once again $x_{1} \cdots x_{n}=0$. Hence, $a_{1}^{q-1} \cdots a_{m n}^{q-1}=0$. This contradicts the minimality of $q$, and hence $q=1$. Therefore, by (22), $a_{1} \cdots a_{m}=0$, and thus $S$ is nilpotent. The converse follows at once from Lemma 1. 
Combined with Corollary 3 , we easily obtain

CoRollary 4. Let $R$ be a commutative associative $\beta_{n}$-ring with Jacobson radical $J$. Then $J$ is nilpotent and $R / J$ is finite.

We conclude with the following two remarks.

REMARK 1. The group-theoretic analogue of Theorem 1 is false. Indeed, the group $Z\left(p^{\infty}\right)$, which consists of the set of all $p^{n}$ th roots of unity, where $p$ is a fixed prime and $n=0,1,2, \cdots\left[3\right.$, p. 4], is a $\beta_{2}$-semigroup. To prove this, suppose that $x_{1}, x_{2} \in Z\left(p^{\infty}\right)$. Then, for some integer $n$, $x_{1}, x_{2} \in Z\left(p^{n}\right)$, where $Z\left(p^{n}\right)$ is the group of all $p^{n}$ th roots of unity. Let $\sigma$ be a generator of $Z\left(p^{n}\right)$. Then

$$
x_{1}=\sigma^{r}, \quad x_{2}=\sigma^{s} ; \quad 1 \leqq r \leqq p^{n}, 1 \leqq s \leqq p^{n} .
$$

Now, let

$$
r=r_{0} p^{i}, \quad s=s_{0} p^{j} ; \quad\left(r_{0}, p\right)=1,\left(s_{0}, p\right)=1,
$$

and suppose, without loss of generality, that $i \leqq j$. Since $\left(r / p^{i}, p\right)=1$, there exists a solution $x$ to

$$
\left(r / p^{i}\right) x \equiv s / p^{j} \bmod p^{n},
$$

and hence $r x p^{j-i} \equiv s \bmod p^{n}$. Thus, $r+s \equiv r\left(1+x p^{j-i}\right) \bmod p^{n}$, and hence

$$
\sigma^{r+s}=\left(\sigma^{r}\right)^{1+x p^{j-i}} \text {, }
$$

since $\sigma^{p^{n}}=1$. Therefore $x_{1} x_{2}=\left(x_{1}\right)^{1+x p^{j-i}}$ and thus $Z\left(p^{\infty}\right)$ is a $\beta_{2}$-semigroup.

Remark 2. The converse of Corollary 4 is false. To see this, let $F$ be an infinite field of characteristic 2 , and let

$$
R=\left\{\left(\begin{array}{ll}
a & x \\
0 & a
\end{array}\right) \mid a \in G F(2), x \in F\right\} .
$$

It is readily verified that the Jacobson radical $J$ of $R$ satisfies $J^{2}=(0)$, and, moreover, $R / J \cong G F(2)$. However, $R$ is not a $\beta_{n}$-ring for any $n$. We prove this by contradiction. Thus, suppose that $R$ is a $\beta_{n}$-ring for some integer $n$, and define

$$
T=\left\{\left(\begin{array}{ll}
1 & x \\
0 & 1
\end{array}\right) \mid x \in F\right\} .
$$

By Lemma 2, $T$ is a $\beta_{n}$-semigroup. Moreover, the mapping

$$
\sigma: x \rightarrow\left(\begin{array}{cc}
1 & x \\
0 & 1
\end{array}\right) \quad(x \in F)
$$

is easily seen to yield an isomorphism of $F(+)$ onto $T(\times)$. But [3, p. 17]

$$
\begin{aligned}
F(+) \cong & \text { a direct sum of an infinite number of } \\
& \text { nontrivial finite cyclic groups } G_{i}(i \in \Gamma) .
\end{aligned}
$$


Now, let $a_{i} \in G_{i} ; a_{i} \neq 0 ; i=1, \cdots, n$, and let the elements $x_{i}$ of $F$ be defined by (see (29))

$$
x_{i}=\left(0,0, \cdots, 0, a_{i}, 0,0, \cdots\right) \quad i=1, \cdots, n,
$$

where $a_{i}$ appears in the $i$ th position. Let $x_{i}^{\prime}$ be the element of $T$ defined by

$$
x_{i}^{\prime}=\left(\begin{array}{cc}
1 & x_{i} \\
0 & 1
\end{array}\right)\left(=\sigma\left(x_{i}\right)\right), \quad i=1, \cdots, n .
$$

Since $T$ is a $\beta_{n}$-semigroup, there exists a word $w=w_{x_{1}{ }^{\prime}, \cdots, x_{n}}\left(x_{1}^{\prime}, \cdots, x_{n}^{\prime}\right)$, with some $x_{j}^{\prime}$ missing in $w$, such that

$$
x_{1}^{\prime} \cdots x_{n}^{\prime}=w_{x_{1}^{\prime}, \cdots, x_{n}}\left(x_{1}^{\prime}, \cdots, x_{n}^{\prime}\right) .
$$

Now, in view of the isomorphism $\sigma$, this equation reflects in $F(+)$ as (see (28) and (31))

$$
x_{1}+\cdots+x_{n}=w^{*}\left(x_{1}, \cdots, x_{n}\right),
$$

where $w^{*}=w^{*}\left(x_{1}, \cdots, x_{n}\right)$ is the (additive) word obtained by replacing " $x$ " by " + " throughout the word $w$, and by replacing each $x_{i}^{\prime}$ by $x_{i}$. Moreover, since $x_{j}^{\prime}$ is missing in $w, x_{j}$ is missing in the word $w^{*}$. Now, equating the $j$ th coordinates of both sides of (33) (see (30)), we get $a_{j}=0$ (since $w^{*}\left(x_{1}, \cdots, x_{n}\right)$ does not involve $\left.x_{j}\right)$, which contradicts the choice of $a_{j}$. This contradiction proves that $R$ is not a $\beta_{n}$-ring.

In conclusion, we wish to express our indebtedness and gratitude to the referee for his valuable suggestions.

\section{REFERENCES}

1. I. N. Herstein, Theory of rings, Lecture Notes, University of Chicago, Chicago, Ill., 1961.

2. N. Jacobson, Structure of rings, rev. ed., Amer. Math. Soc. Colloq. Publ., vol. 37, Amer. Math. Soc., Providence, R.I., 1964. MR 36 \#5158.

3. I. Kaplansky, Infinite abelian groups, Univ. of Michigan Press, Ann Arbor, Mich., 1954. MR 16, 444.

4. T. Nagell, Introduction to number theory, 2nd ed., Chelsea, New York, 1964. MR 30 \#4714.

5. H. Rademacher, Lectures on elementary number theory, Blaisdell, Waltham, Mass., 1964. MR 30 \#1079.

6. B. L. van der Waerden, Elementarer Beweis eines zahlentheoretischen Existenztheorems, J. Reine Angew. Math. 171 (1934), 1-3.

Department of Mathematics, University of California, Santa Barbara, CaLifornia 93106 